\title{
HARMONIC MEASURES SUPPORTED ON CURVES
}

\author{
C. J. Bishop, L. Carleson, J. B. Garnett and P. W. Jones
}

Let $\Omega_{1}$ and $\Omega_{2}$ be two disjoint, simply connected domains in the plane, and let $\omega_{1}$ and $\omega_{2}$ be harmonic measures associated to $\Omega_{1}$ and $\Omega_{2}$. We present necessary and sufficient conditions for $\omega_{1}$ and $\omega_{2}$ to be mutually singular.

1. Introduction. Let $\Gamma$ be a Jordan curve in $C$ and let $\Omega_{1}$ and $\Omega_{2}$ be the two simply connected domains complementary to $\Gamma$. For each domain $\Omega_{j}$ fix a point $z_{j} \in \Omega_{j}$ and let $\omega_{j}$ be the harmonic measure for $z_{j}$ relative to $\Omega_{j}$. In this paper we discuss when the two measures are singular, $\omega_{1} \perp \omega_{2}$, i.e. when there are disjoint sets $E_{1}, E_{2}$ such that $\omega_{j}\left(E_{j}\right)=1, j=1,2$. If $\Gamma$ is a Jordan arc, $\Gamma^{c}$ consists of only one domain $\Omega$, but since $\Gamma$ has two sides there are two measures $\omega_{1}, \omega_{2}$ which give the harmonic measure of sets on each of the two sides of $\Gamma$. Again it makes sense to ask whether $\omega_{1} \perp \omega_{2}$.

If $\Gamma$ is a Jordan curve or arc and $z_{0} \in \Gamma$ we say that $\Gamma$ has a tangent at $z_{0}$ if there is $\theta_{0}$ with the property that for all $\varepsilon>0$ there is $r>0$ such that whenever $z \in \Gamma$ and $\left|z-z_{0}\right|<r$, either $\left|\theta_{0}-\arg \left(z-z_{0}\right)\right|<\varepsilon$ or $\left|\theta_{0}+\pi-\arg \left(z-z_{0}\right)\right|<\varepsilon$. We denote by $T$ the collection of all tangent points on $\Gamma$. When $\Gamma$ is a Jordan curve we also say that $z_{0} \in T_{1}$ if there is a unique $\theta_{0}(\bmod 2 \pi)$ with the property that for all $\varepsilon>0$ there is $r>0$ such that

$$
\left\{z: 0<\left|z-z_{0}\right|<r,\left|\theta_{0}-\arg \left(z-z_{0}\right)\right|<\pi / 2-\varepsilon\right\} \subset \Omega_{1} .
$$

$T_{1}$ is called the set of inner tangent points with respect to $\Omega_{1}$. With $T_{2}$ similarly defined one sees that $T=T_{1} \cap T_{2}$. If $\Gamma$ is a Jordan arc $T_{1}$ and $T_{2}$ are similarly defined. Finally, we denote one dimensional Hausdorff measure by $\Lambda_{1}$.

THEOREM. Suppose $\Gamma$ is a Jordan curve or arc. Then $\omega_{1} \perp \omega_{2}$ if and only if $\Lambda_{1}(T)=0$.

Let $A(\Gamma)$ denote the class of all bounded continuous functions on the Riemann sphere which are holomorphic off $\Gamma$. In [4] Browder and Wermer proved that $A(\Gamma)$ is a Dirichlet algebra if and only if $\omega_{1} \perp \omega_{2}$. 
Corollary. $A(\Gamma)$ is a Dirichlet algebra if and only if $\Lambda_{1}(T)=0$.

We prove the theorem in $\S 2$ and make some remarks in $\S 3$.

2. Proof of the theorem. We prove the theorem in the case where $\Gamma$ is a Jordan curve; the modifications needed when $\Gamma$ is an arc are outlined in $\S 3$. First suppose that $\Lambda_{1}(T)>0$. It is then an easy matter to find two curves $\Gamma_{1}, \Gamma_{2}$ such that each $\Gamma_{j}$ is rectifiable, $\Gamma_{j} \subset \bar{\Omega}_{j}$, and $\Lambda_{1}\left(\Gamma_{1} \cap \Gamma_{2} \cap T\right)>0$. Denoting by $\tilde{\Omega}_{j}$ the component of $\Gamma_{j}^{c}$ contained in $\Omega_{j}$, we may also assume that $z_{j} \in \tilde{\Omega}_{j}, j=1,2$. Let $\tilde{\omega}_{1}, \tilde{\omega}_{2}$ be the obvious associated harmonic measures, and let $E=\Gamma_{1} \cap \Gamma_{2} \cap T$. Since $\Gamma_{j}$ is rectifiable, $\tilde{\omega}_{j}$ is mutually absolutely continuous with respect to $\Lambda_{1}, \tilde{\omega}_{j} \ll \Lambda_{1} \ll \tilde{\omega}_{j}$, and consequently $\tilde{\omega}_{1}(E), \tilde{\omega}_{2}(E)>0$. But by the maximum principle, $\tilde{\omega}_{j}(E) \leq \omega_{j}(E), j=1,2$. We have thus proven that if $\Lambda_{1}(T)>0$, it cannot be that $\omega_{1} \perp \omega_{2}$.

We now assume that $\Lambda_{1}(T)=0$ and make the normalizing assumption distance $\left(z_{j}, \Gamma\right) \geq 1, j=1,2$.

Lemma 1. Suppose $z_{0} \in \Gamma$ and $D=\left\{z:\left|z-z_{0}\right| \leq r\right\}$ where $r<1$. Then

$$
\omega_{1}(D \cap \Gamma) \cdot \omega_{2}(D \cap \Gamma) \leq A r^{2}
$$

where $A$ is independent of $z_{0}, \Gamma$ and $r$.

Proof. This lemma should be credited to Beurling; it is contained in the last section of his thesis [2]. For completeness we include a proof. Without loss of generality the component $\Omega_{1}$ is bounded. The set $\Gamma \backslash D$ can be written as a disjoint collection of open arcs $\gamma_{k}$. For exactly one of these arcs $\gamma_{k}$, call it $\gamma$, it is true that $C \backslash\{\gamma \cup D\}$ has a bounded component, call it $\hat{\Omega}_{1}$, containing $z_{1}$. Let $\hat{\Omega}_{2}$ denote the component of $\mathbf{C} \backslash\{\gamma \cup D\}$ containing $z_{2}$, and let $\hat{\omega}_{j}$ be the harmonic measures associated to $\hat{\Omega}_{j}$ and $z_{j}, j=1,2$. Then by the maximum principle,

$$
\omega_{j}(D \cap \Gamma) \leq \hat{\omega}_{j}\left(D \cap \partial \hat{\Omega}_{j}\right), \quad j=1,2 .
$$

Fix $t, r<t<1$, and let $\gamma_{1}(t)$ be the unique subarc of $\left\{z \in \hat{\Omega}_{1}:\left|z-z_{0}\right|=\right.$ $t$ ) which separates $D$ from $z_{1}$ in $\hat{\Omega}_{1}$. Let $\theta_{1}(t)=\Lambda_{1}\left(\gamma_{1}(t)\right)$. Define in a similar fashion $\gamma_{2}(t)$ and $\theta_{2}(t)$ with respect to the domain $\hat{\Omega}_{2}$. The distortion theorem (see e.g. pp. 76-78 of [1]) asserts that

$$
\hat{\omega}_{j}\left(D \cap \partial \hat{\Omega}_{j}\right) \leq A \exp \left\{-\pi \int_{r}^{1} \frac{d t}{\theta_{j}(t)}\right\}, \quad j=1,2 .
$$


Since $\theta_{1}(t)+\theta_{2}(t) \leq 2 \pi t$, inequality (1) yields

$$
\begin{aligned}
& \omega_{1}(D \cap \Gamma) \cdot \omega_{2}(D \cap \Gamma) \\
& \leq A^{2} \exp \left\{-\pi \int_{r}^{1} \frac{2 d t}{\pi t}\right\} \leq A^{2} r^{2} .
\end{aligned}
$$

In [6] Makarov developed an ingenious and simple method using Plessner's theorem to show that whenever $\Omega$ is simply connected there is a set $E$ of full harmonic measure and Hausdorff dimension one. We shall use a slightly sharper version of that result which has been obtained by Pommerenke [8]. Let $\Omega$ be a Jordan domain and let $\omega$ be harmonic measure with respect to $\Omega$. Let $E$ be the collection of all inner tangents with respect to $\Omega$ and let $F=\partial \Omega \backslash E$. Then Pommerenke shows that with $\left.\omega^{a} \equiv \omega\right|_{E}$ and $\left.\omega^{s} \equiv \omega\right|_{F}$ one has

$$
\omega^{a} \ll \Lambda_{1} \ll \omega^{a} \text { on } E
$$

and

(3) For all $M, r_{0}>0$ there are disks $D_{k}=D\left(\zeta_{k}, r_{k}\right)$ where $r_{k}<r_{0}$,

$$
\omega^{s}\left(\bigcup_{k} D_{k}\right)=\omega^{s}(F) \text {, and } \omega^{s}\left(D_{k}\right) \geq M r_{k} \text {. }
$$

Let $\omega_{j}^{a}=\left.\omega_{j}\right|_{T_{j}}$ and let $\omega_{j}^{s}=\omega_{j}-\omega_{j}^{a}, j=1,2$. Then since $\Lambda_{1}(T)=$ $\Lambda_{1}\left(T_{1} \cap T_{2}\right)=0$, condition (2) shows that $\omega_{1}^{a} \perp \omega_{2}^{a}$. On the other hand, taking $M$ large and applying (3) yields $\omega_{1}^{a} \perp \omega_{2}^{s}$ and $\omega_{1}^{s} \perp \omega_{2}^{a}$. It is therefore only necessary to prove $\omega_{1}^{s} \perp \omega_{2}^{s}$. To this end notice by (3) that there is a set $\tilde{F}$ such that $\omega_{1}^{s}(\tilde{F})=\left\|\omega_{1}^{s}\right\|$ and such that for all $z \in \tilde{F}$ there are disks $D_{n} \downarrow z$ such that $z \in D_{n}$ and

$$
\omega_{1}^{s}\left(D_{n}\right) \geq M r_{n},
$$

where $r_{n}$ is the radius of $D_{n}$. But by Lemma 1 ,

$$
\omega_{1}^{s}\left(D_{n}\right) \cdot \omega_{2}^{s}\left(D_{n}\right) \leq A r_{n}^{2} .
$$

Taking $M$ larger, we see that (4) and (5) imply $\omega_{1}^{s} \perp \omega_{2}^{s}$.

3. Remarks. When $\Gamma$ is not a curve but an arc, the only point that needs modification in the preceding proof is that in Lemma 1 the conclusion must be weakened to $\omega_{1}(D \cap \Gamma) \cdot \omega_{2}(D \cap \Gamma) \leq A_{z_{0}} r^{2}$, where $A_{z_{0}}$ depends on $z_{0}$, and one also requires that $r \leq r_{z_{0}}=$ $\min \left\{\left|z_{0}-\zeta_{1}\right|,\left|z_{0}-\zeta_{2}\right|\right\}$, where $\zeta_{1}$ and $\zeta_{2}$ are the two endpoints of $\Gamma$. That is because the distortion theorem can only be used to conclude

$$
\hat{\omega}_{J}(D \cap \partial \hat{\Omega}) \leq A \exp \left\{-\pi \int_{r}^{r_{z_{0}}} \frac{d t}{\theta_{j}(t)}\right\} .
$$


Here $\hat{\Omega}$ is the appropriate domain formed out of $\Gamma$ and $D$.

The theorem can be generalized to the case where $\Gamma$ is not a Jordan curve. Let $\Omega_{1}$ and $\Omega_{2}$ be two disjoint, simply connected domains and denote by $T_{1}$ and $T_{2}$ the respective sets of inner tangent points. Then $\omega_{1} \perp \omega_{2}$ if and only if $\Lambda_{1}\left(T_{1} \cap T_{2}\right)=0$. The proof of Lemma 1 is then most easily accomplished by the previous argument together with Beurling's theorem: $\omega(E) \leq C \exp \{-\pi \lambda\}$ where $\lambda$ is the extremal length associated to all paths in a domain $\Omega$ joining some disk $K \Subset \Omega$ to $E \subset \partial \Omega$. (See [7] for an alternative proof.) A minor modification of the theorem can also be used to prove $\omega_{1} \ll \omega_{2} \ll \omega_{1}$ if and only if for all $\varepsilon>0$ there are rectifiable curves $\Gamma_{j} \subset \bar{\Omega}_{j}$ such that $\omega_{j}\left(\Gamma_{1} \cap \Gamma_{2}\right)>1-\varepsilon, j=1,2$.

It is worth noting that previous authors (see e.g. [5]) have used the Browder-Werner theorem to conclude in certain cases $\omega_{1} \perp \omega_{2}$. An interesting problem that remains open is to construct one non constant function in $A(\Gamma)$ for a general arc $\Gamma$ where $\omega_{1} \perp \omega_{2}$.

Added in Proof. See [3] for a construction of non constant functions in $A(\Gamma)$.

\section{REFERENCES}

[1] L. V. Ahlfors, Conformal Invariants, McGraw-Hill, Inc., 1973.

[2] A. Beurling, Études sur un problème de majoration, thesis, Upsal (Uppsala), 1933.

[3] C. J. Bishop, Constructing continuous functions holomorphic off a curve, J. Funct. Anal., 82 (1989), 113-137.

[4] A. Browder and J. Wermer, Some algebras of functions on an arc, J. Math. Mech., 12 (1963), 119-130.

[5] T. W. Gamelin and J. B. Garnett, Pointwise bounded approximation and Dirichlet algebras, J. Funct. Anal., 8 (1971), 360-404.

[6] N. G. Makarov, On the distortion of boundary sets under conformal mappings, Proc. London Math. Soc. (3), 51 (1985), 369-384.

[7] A. Pfluger, Extremallängen und Kapazität, Comm. Math. Helv., 29 (1955), 120-131.

[8] Ch. Pommerenke, On conformal mapping and linear measure, preprint.

Received September 1, 1987.

UNIVERSITY OF CALIFORNIA

LOS ANGELES, CA 90024-1555-05

AND

YALE UNIVERSITY

New Haven, CT 06520 